\title{
AGRICULTURA ORGÁNICA Y PARTICIPACIÓN CAMPESINA EN EL CENTRO UNIVERSITARIO DE LA COSTA SUR DE LA UNIVERSIDAD DE GUADALAJARA, MÉXICO
}

\section{ORGANIC AGRICULTURE AND RURAL PARTICIPATION AT THE SOUTHERN COAST OF JALISCO UNIVERSITY CENTER, UNIVERSITY OF GUADALAJARA, MEXICO}

\author{
Víctor Manuel Villalvazo López* \\ Pedro Figueroa Bautista** \\ Gerardo Cruz Sandoval ${ }^{* * *}$ \\ Víctor Manuel Sánchez Bernal**** \\ José Luis Olguín López ${ }^{* * * * *}$
}

RESUMEN

\begin{abstract}
Este artículo analiza el rol que tienen los talleres de agricultura campesina en el diseño de estrategias para la promoción del diálogo, el empoderamiento y la educación popular campesina, en el periodo 2010-2019, en el Centro Universitario de la Costa Sur (cucsur) de la Universidad de Guadalajara. El enfoque metodológico es de tipo cualitativo, con aportes de la investigación participativa. Las técnicas utilizadas son la realización de talleres teóricosprácticos, donde los campesinos "aprenden haciendo". Los talleres resultaron ser espacios adecuados no solo para generar tecnologías agrícolas apropiadas, sino para construir alternativas de comunicación y de educación.
\end{abstract}

\footnotetext{
* Universidad de Guadalajara, Departamento de Ecología y Recursos Naturales-IMEcBIo, Autlán de Navarro, Jalisco, México. vvillalv@cucsur.udg.mx

** Universidad de Guadalajara, Departamento de Ecología y Recursos Naturales-IMEcBIo, Autlán de Navarro, Jalisco, México. pfigueroa@cucsur.udg.mx

*** Universidad de Guadalajara, Departamento de Ecología y Recursos Naturales-IMEcBIo, Autlán de Navarro, Jalisco, México. gerardo.cruz@cucsur.udg.mx

**** Universidad de Guadalajara, Departamento de Ecología y Recursos Naturales-IMECBIo, Autlán de Navarro, Jalisco, México. vsanchezmetate@gmail.com

****** Universidad de Guadalajara, Departamento de Ecología y Recursos Naturales-IMECBio, Autlán de Navarro, Jalisco, México. olguin@cucsur.udg.mx
} 
PALABRAS CLAVE: EDUCACIÓN POPULAR * SABERES CAMPESINOS * AGRICULTURA ORGÁNICA
$*$ EMPODERAMIENTO * PARTICIPACIÓN CAMPESINA

\section{ABSTRACT}

This article analyzes the role that rural agriculture workshops play in designing strategies for dialogue promotion, empowerment and rural popular education, from 2010 to 2019, at the Southern Coast of Jalisco University Center (cucsur in Spanish) of the University of Guadalajara. The methodological approach is qualitative, with contributions from participatory research. The techniques used are the realization of theoretical and practical workshops, where the country people "learn by doing". The workshops proved to be adequate spaces not only to generate appropriate agricultural technologies, but to build communication and education alternatives.

KEYWORDS: POPULAR EDUCATION * RURAL KNOWLEDGE * ORGANIC AGRICULTURE * EMPOWERMENT * COUNTRY PEOPLE PARTICIPATION

\section{INTRODUCCIÓN}

Los problemas globales socioambientales que viven los seres humanos en la actualidad se conciben como resultado del desarrollo de la visión eurocéntrica como único modelo de vida posible en el mundo. Esto trajo como consecuencia la deslegitimación de los saberes y de las vivencias culturales que no son propios de este modelo, con lo cual se establecieron, de manera progresiva, los paradigmas dominantes respecto al significado de "mundo civilizado" (de Sousa Santos, 2010; de la Cuadra, 2015).

Por esta razón, varios de los problemas que actualmente se vive en las regiones rurales del país se deben, en gran parte, a que México - como otros tantos países de Latinoaméricadecidió tomar como ruta de crecimiento este enfoque, cuyos principios fueron la base ideológica del llamado modelo económico neoliberal.

Bajo estos principios se promovió la modernización y la tecnificación del campo en el entorno rural, lo cual transformó los paisajes primarios del territorio geográfico, así como, las estrategias productivas de los campesinos (Calva, 1993; Saxe-Fernández, 1999).

A partir de estas políticas de desarrollo, se permearon los diversos saberes y conocimientos de los grupos de indígenas y campesinos de América Latina, y particularmente de México, que terminaron por ser excluidos de las políticas dirigidas al sector agropecuario. Sin embargo, desde hace aproximadamente
4 décadas han surgido nuevas ideas sobre cómo entender el mundo y su parte social de manera diferente al concepto eurocéntrico. Nociones como el "Buen vivir", el "Desarrollo Endógeno", "El Diálogo de Saberes", niegan los principios rectores economicistas del modelo económico neoliberal y abunda en valores como: derechos, democracia, justicia, diversidad cultural, el uso de recursos y saberes locales, la interculturalidad entre los conocimientos para reivindicar las sabidurías y las diversas formas locales de organización en la búsqueda del otro desarrollo - el de los pueblos originarios- (Roncal, 2017; Loera-González, 2015).

Lo que caracteriza a esta cosmovisión indígena es la postura abierta e incluyente sobre otras formas de generación de conocimiento y respeto a la universalidad de los saberes, entre ellos, los relacionados con las diversas maneras de hacer agricultura por los campesinos tradicionales.

Esta cosmovisión de enfoques se encuentra implícita en el desarrollo de la agricultura orgánica y en los espacios sociales y geográficos en los que se lleva a cabo, donde la participación y conocimientos locales vinculados a las ciencias de la agricultura son fundamentales para su desarrollo.

En el presente estudio se recuperan las experiencias de un programa de Agricultura Orgánica, donde un grupo de campesinos y productores del Occidente de Jalisco, así como estudiantes de la carrera de Ingeniería en Recursos Naturales y Agropecuarios (IRNA), en 
el Centro Universitario de la Costa Sur de la Universidad de Guadalajara (cucsur de la U. de G.) compartieron conocimientos, experiencias y necesidades de lucha social para el desarrollo $y$ la promoción de la agricultura campesina.

Estas acciones son parte del trabajo de Investigación-Acción que se lleva a cabo en el Laboratorio de Desarrollo Rural del Departamento de Ecología y Recursos Naturales-Instituto Manantlán de Ecología y Conservación de la Biodiversidad (DERN-IMECBIO) del CUCsur de la Universidad de Guadalajara donde se han mantenido relaciones de vinculación institucional en la región durante más de 20 años y se consolidan alternativas productivas vinculadas al quehacer campesino en el Occidente de Jalisco (Villalvazo-López et al., 2003; Gerritsen et al., 2004; Figueroa et al., 2005; Gerritsen et al., 2012).

El objetivo de este proyecto es la sistematización de experiencias para el desarrollo de estrategias alternativas de comunicación y educación popular centradas en la participación de académicos y de productores campesinos.

\section{LA NECESIDAD DE LA EDUCACIÓN POPULAR}

Las carencias y las necesidades de las clases sociales oprimidas como las que enfrenta la población campesina son las que deberían generar la conciencia colectiva institucional $y$ gubernamental necesaria para contribuir a la resolución de la problemática del campo, apoyados por las instancias educativas no solo de México, sino de todo el mundo. Sin embargo, para lograr esto se requiere desaprender los conceptos eurocentristas que sustentan que la cultura, los valores, $y$ las tradiciones europeas son superiores a las propias de Latinoamérica, $y$ que de manera histórica se han impartido en el modelo educativo del país. En este caso en particular, se recomienda la revisión de la currícula de las ciencias agronómicas que se ofertan en las universidades mexicanas. Con algunas excepciones, se encuentra que los contenidos temáticos están fuertemente dirigidos a cubrir las necesidades comerciales de las empresas de agroquímicos, no de las necesidades productivas de las personas campesinas.
Esto, sin duda, es una de las causas de la dependencia tecnológica histórica que la población campesina tiene con las empresas. Esto es sin duda uno de los roles que las universidades deben retomar como parte de sus acciones sustantivas para la generación y transferencia de conocimiento a las comunidades campesinas. Además, esta acción debe ser funcional para la localidad donde estas se desarrollan pues, de lo contrario, lo que se oferta en las universidades no está contribuyendo a generar prácticas transformadoras para los pueblos (Kaplun, 1985).

La generación de espacios plurales para la generación de conocimientos, más allá del simple desarrollo del método científico, y por supuesto de estrategias alternativas de comunicación $y$ de metodologías educativas, son necesarias en la actualidad, puesto que son fundamentales para fomentar el diálogo y el intercambio de conocimientos empíricos y científicos.

Históricamente, se ha reconocido al campesinado por su valioso bagaje cultural de experiencias y conocimientos empíricos, lo cual los convierte en actores primarios en la difusión de los saberes locales para sus hijos e hijas con aspiraciones profesionales. Retomar los conocimientos que los hijos y las hijas de esta población traen de sus comunidades es fundamental, pues son una parte implícita de la memoria biocultural presente en las comunidades donde se conjugan las experiencias, el ingenio y la creatividad para el desarrollo de la agricultura campesina (Toledo y Barrera-Bassols, 2008).

Esta integración habría de considerarse como una estrategia de revolución educativa para las universidades, la cual transforme a eruditos e intelectuales del campo de las ciencias sociales $y$ naturales en verdaderos agentes de cambio social.

El objetivo final es recuperar y promover el enorme acervo cultural que existe en la palabra y los saberes campesinos para el manejo sustentable de los recursos naturales. Se debe considerar que el conocimiento generado fuera de las universidades se vuelve "los otros saberes", contrario a aquel conocimiento vertical, unidireccional, monopólico y al servicio 
de las minorías; por lo tanto, este no puede transformarse en una práctica transformadora (Kaplun, 1985).

Por esta razón, resulta incongruente que la formación profesional para el desarrollo del campo se vuelva en sí misma un círculo de comunicación cerrado de intelectuales y empresarios agropecuarios.

De aquí se deriva la necesidad de que los profesionistas del agro adquieran conciencia de que es posible inmiscuirse en "la otra comunicación", la alternativa, que surge como mecanismo de defensa hacia el conocimiento dominante, la que no manipula ni excluye. Una vez que ocurra esto, podrán surgir los nuevos protagonistas para la aplicación de novedosos esquemas de aprendizajes en la relación histórica pueblo-universidad o campesino-universidad.

Dicho lo anterior, se requiere un concepto de la comunicación que integre dichas estrategias. Para esto, el término de "alternativas de comunicación" es útil pues se manifiesta como un arma de lucha ideológica que se entiende como una "expresión sistemática, coherente, creativa, complementaria, abierta y horizontal, que intercomunica el nivel de conciencia de un grupo social específico, su avance organizativo y sus luchas" (Núñez, 1985). Aquí surge la pregunta: ¿qué proyección debe dársele a ese proceso de comunicación para enfocar estos conocimientos? Es un hecho que deberá ser una proyección multisentido que, reconozca, por un lado, que los conocimientos generados deberán fortalecer la conciencia social de aquellos grupos que han sido oprimidos y relegados de los modelos de desarrollo desde hace cientos de años $y$, por otro lado, este conocimiento sirva de detonante para iniciar procesos de cambio social. Evidencias significativas de esta conceptualización son visibles en las manifestaciones y expresiones de luchas sociales, que han sido comunes en la historia de Latinoamérica.

En esta gran región se han desarrollado -y continúan gestándose en la actualidadgrandes fenómenos revolucionarios que ponen en debate los efectos negativos por la imposición única del conocimiento dominante. Esto, a su vez, ha impulsado a cientos de grupos sociales en la construcción de nuevas visiones de desarrollo, no sustentados necesariamente en las perspectivas y los paradigmas de los modelos de educación occidental (Roncal, 2017).

Por todo lo anterior, resulta indispensable repensar el papel que pueden jugar las instituciones educativas de educación agronómica en la generación de nuevas visiones o nuevos enfoques educativos para la acción social, como es el caso de la presente investigación.

En este sentido, se intenta comprender las percepciones de un grupo de campesinos y estudiantes con base al quehacer cotidiano de la agricultura, en un estudio de caso vinculado al desarrollo de talleres de agricultura orgánica con participación de campesinos, estudiantes $y$ académicos.

\section{MATERIALES Y MÉTODOS}

\section{A) ÁREA DE ESTUDIO}

El estudio se desarrolló en el cucsur de la Universidad de Guadalajara, ubicado en la cabecera municipal de Autlán de Navarro Jalisco, México, entre las coordenadas $19^{\circ} 46^{\prime} 20^{\prime \prime}$ y $19^{\circ} 46^{\prime} 37^{\prime \prime}$ de latitud norte, y $104^{\circ} 21^{\prime} 24^{\prime \prime}$ y $104^{\circ} 21^{\prime} 45^{\prime \prime}$ de longitud oeste a una altitud de $920 \mathrm{msnm}$ (figura 1). 
Figura 1

Localización del CUCSUR de la Universidad de Guadalajara

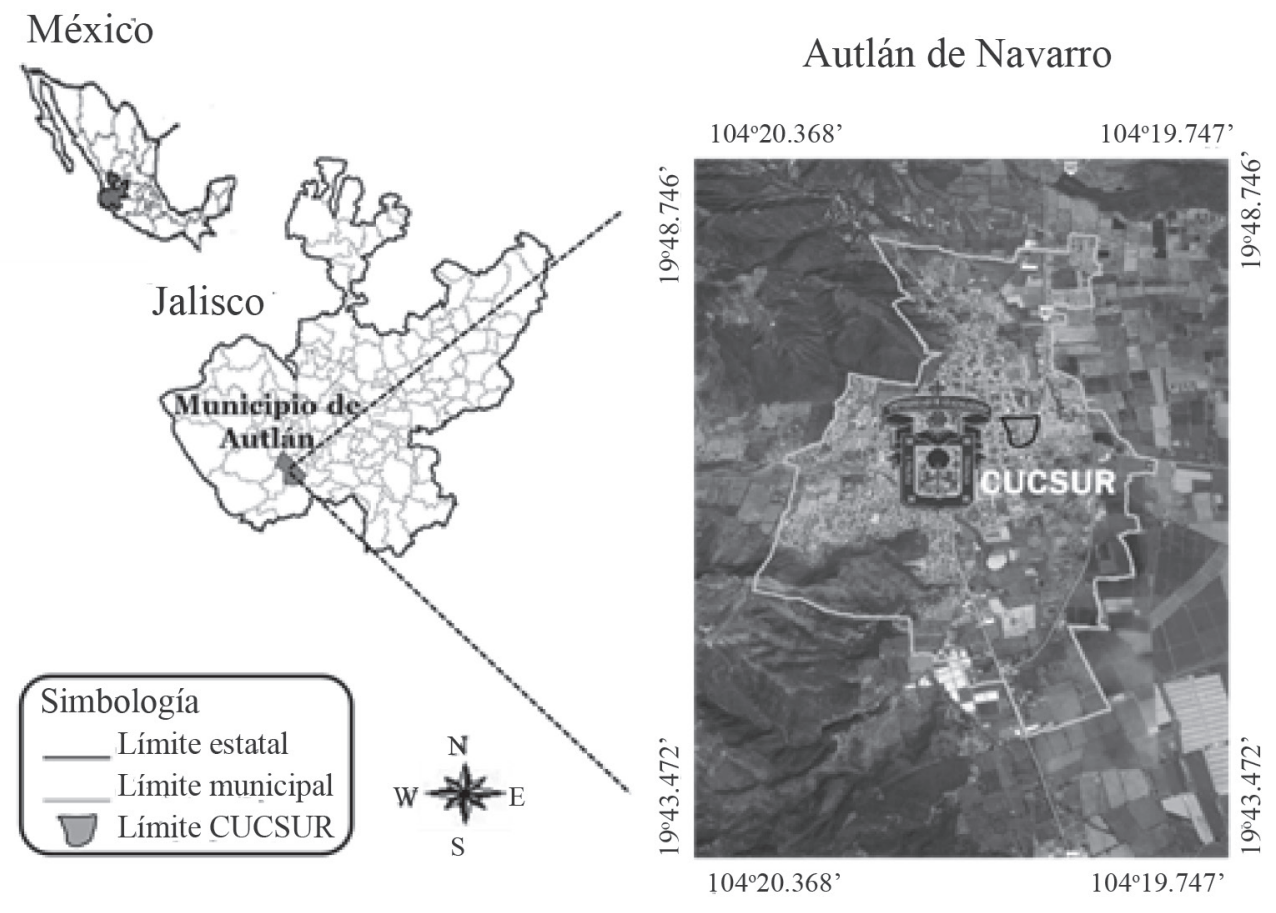

Nota: Elaboración propia a partir del InEgi, Marco Geoestadístico, diciembre 2018 y Google Earth fragmentado, noviembre de 2019. Imagen Landsat/copernicus.

Los talleres educativos de agricultura orgánica se imparten como una actividad de la currícula de las unidades de aprendizaje del curso Agricultura Orgánica del plan de estudios de la carrera IRNA, utilizando la infraestructura y las aulas del cucsur. Estas se llevan cabo en módulos semestrales durante cada ciclo escolar semestral, donde se desarrollan dos horas de teoría y dos de práctica los viernes de cada semana, de conformidad al calendario escolar de la Universidad de Guadalajara.

Lo que hace diferente a otros cursos impartidos de la carrera de IRNA, es que son espacios situacionales educativos de participación de estudiantes, campesinos y técnicos facilitadores y profesores (sin exclusión de género), los cuales acuden tanto para coparticipar en la difusión y desarrollo de las tecnologías, como para iniciar procesos de reflexión-aprendizaje en la búsqueda de alternativas viables para el desarrollo de la agricultura orgánica.

El énfasis relevante de los talleres es despertar la curiosidad y alentar la creatividad de las personas participantes para el desarrollo de la agricultura orgánica en su localidad, ya que se trabaja con grupos diversos y en diferentes condiciones. La temática se enfoca a las necesidades situacionales de cada localidad.

\section{MÉTODOS}

Se utilizó la metodología cualitativa con aplicación de muestreo intencional dirigido a participantes voluntarios por invitación (auto-seleccionados) con un número de 16 entrevistas (Levin, 
1992). De estas personas, 8 son "campesinos productores" ( 3 mujeres y 5 hombres) y 8 son "estudiantes" (4 mujeres y 4 hombres) de la carrera de IRNA. El eje central de la unidad de análisis es los "campesinos productores y los estudiantes”, junto con sus saberes y percepciones sociales.

El criterio de selección de los "campesinos productores", fue la asistencia de al menos cuatro módulos semestrales de los talleres de agricultura orgánica ofertados en el CUCSUR y que se encuentran desarrollando acciones de agricultura orgánica en sus predios o parcelas. Por su parte, el criterio de selección de "estudiantes", fue ser alumno o alumna regular del curso de agricultura orgánica y contar con una participación activa en prácticas y en asistencia continua al curso. Se aplicó entrevistas semiestructuradas y se analizó su discurso estableciendo categorías, utilizando técnicas de la metodología cualitativa. Para este proyecto en específico, se permite a los productores y las productoras nombrar al cucsuR, de manera equivalente al de la universidad, al considerar de antemano que se refieren al cucsur, sin demeritar a la Universidad de Guadalajara como institución.

Las entrevistas dan respuesta a las siguientes variables: demográficas, de vinculación regional de proyectos de investigación agropecuaria $y$, finalmente, de saberes y prácticas agrícolas (tabla 1$)$.

Tabla 1

Variables de análisis de percepciones de "personas productoras campesinas" y "estudiantes"

\begin{tabular}{|c|c|}
\hline FACTOR & ACTORES Y VARIABLES \\
\hline \multirow{2}{*}{ Demográficas. } & $\begin{array}{l}\text { Productores campesinos: edad, tiempo dedicado a la agricultura, lugar de origen (es relevante } \\
\text { por la lejanía del lugar de origen para trasladarse al cucsuR), grado de estudios (importante para } \\
\text { analiza la adopción y replicabilidad de metodologías y creación de material de aprendizaje). }\end{array}$ \\
\hline & $\begin{array}{l}\text { Estudiantes: no caracterizados por considerarse participantes eventuales, y porque la edad y } \\
\text { nivel de escolaridad son relativamente homogéneos. }\end{array}$ \\
\hline $\begin{array}{l}\text { Vinculación regional } \\
\text { de proyectos de } \\
\text { investigación } \\
\text { agropecuaria. }\end{array}$ & $\begin{array}{l}\text { Productores campesinos y estudiantes: se analiza en ambos la percepción sobre la vinculación } \\
\text { regional de proyectos de investigación agropecuaria, considerando: a) el distanciamiento } \\
\text { entre el cuerpo de conocimientos entre los ingenieros y los campesinos; b) la generación } \\
\text { de conocimiento y publicaciones accesibles a la población campesina; c) el cucsur, como } \\
\text { institución educativa que tenga programas para formar profesionistas en el área agrícola } \\
\text { que respondan a las necesidades campesinas de bajos recursos y d) las percepciones sobre la } \\
\text { utilidad de la información generada en al área agropecuaria para la solución de problemas en } \\
\text { el entorno regional. }\end{array}$ \\
\hline \multirow{3}{*}{$\begin{array}{l}\text { Saberes y prácticas } \\
\text { locales. }\end{array}$} & $\begin{array}{l}\text { Productores campesinos y estudiantes: percepciones sobre las prácticas y saberes locales. } \\
\text { Se examinan los saberes locales (respecto a la agricultura), y su relación con el grado de } \\
\text { inclusión de estos, en la dinámica educativa de cursos y programas vinculados a la enseñanza } \\
\text { de la agricultura en los que los actores han participado. Finalmente, se analiza las diferentes } \\
\text { concepciones de la variable "Tierra" como símbolo cultural y no solo como espacio de } \\
\text { producción. }\end{array}$ \\
\hline & $\begin{array}{l}\text { Estudiantes: para el caso de las personas estudiantes se analizan prácticas y saberes locales, } \\
\text { cuáles se han forjado como parte de la cultura de su lugar de origen, tratando de entender } \\
\text { ¿cómo? o ¿por qué? (en su caso), son desplazados total o parcialmente cuando ellos ingresan } \\
\text { al cucsuR. }\end{array}$ \\
\hline & Productores campesinos: valorización de los saberes en los cursos de agricultura. \\
\hline
\end{tabular}




\section{RESULTADOS}

\section{A) DEMOGRÁFICAS}

Las personas productoras son procedentes de cinco municipios aledaños al cucsur (ciudad de Autlán). De ellos, quien vive más cerca se traslada en aproximadamente veinte minutos desde su lugar de origen; quien vive más lejos tarda aproximadamente tres horas a los cursos. De los productores, cuatro solo tienen el grado de educación primaria básica, uno no concluida $y$ dos que tienen educación superior; así mismo, uno de educación secundaria. Estos datos tendrán relevancia posterior para explicar la importancia de asegurar que existan metodologías apropiadas para lograr un adecuado proceso de comunicación aun sin altos niveles de educación oficial (tabla 2).

Tabla 2

Caracterización de las personas campesinas

\begin{tabular}{cccccc}
\hline $\begin{array}{c}\text { PARTICIPANTE } \\
\text { O } \\
\text { PRODUCTOR }\end{array}$ & $\begin{array}{c}\text { EDAD } \\
\text { (AÑN) }\end{array}$ & $\begin{array}{c}\text { EDAD DE } \\
\text { INCORPORACIÓN } \\
\text { A LA AGRICULTURA }\end{array}$ & $\begin{array}{c}\text { LUGAR DE } \\
\text { ORIGEN }\end{array}$ & $\begin{array}{c}\text { TIEMPO DE } \\
\text { TRASLADO AL } \\
\text { CUCSUR } \\
\text { (VEHÍCULO } \\
\text { PARTICULAR) }\end{array}$ & ESCOLARIDAD \\
\hline 1 & 65 & Desde los 10 años & Tomatlán & 3 hrs. & Primaria \\
1 & 60 & Desde los 10 años & El Grullo & 30 min. & Primaria \\
1 & 58 & Desde los 28 años & La Huerta & 1 hrs. & Primaria sin concluir \\
1 & 72 & Desde los 17 años & Tomatlán & 3 hrs. & Primaria \\
1 & 48 & Desde los 28 años & Autlán & 20 min. & Primaria \\
1 & 69 & Desde los 12 años & Autlán & 20 min. & Superior \\
1 & 68 & Desde los 13 años & Autlán & 20 min. & Superior \\
1 & 32 & Desde los 17 años & Unión de Tula & 40 min. & Secundaria \\
\hline
\end{tabular}

La caracterización de las personas productoras respecto a las variables que se muestran en la tabla 2 resultan básicos para que el lector o lectora desarrolle sus propios razonamientos preliminares sobre lo complejo que resulta el trabajo de educación con universitarios y campesinos que presentan tantas diferencias entre las variables mencionadas. A continuación, se presentan las percepciones sobre la vinculación universidad-sociedad.

\section{B) VINCULACIÓN REGIONAL DE PROYECTOS DE INVESTIGACIÓN AGROPECUARIA}

Se encontró que el $100 \%$ de los participantes demandan proyectos de vinculación orientados a crear un programa de agricultura orgánica, en el cual la Universidad de Guadalajara se interese por los problemas inherentes a la agricultura de la región y se enfoque a resolverlos. Para esto se hizo una integración de categorías propias de acuerdo al indicador social de percepción expresada por la población participante (tabla 3 ). 
Tabla 3

Percepciones del grupo autoseleccionado participante

Escuela Campesina en el Centro Universitario de la Costa Sur

Universidad Guadalajara, 2010-2019

VARIABLE: VINCULACIÓN REGIONAL DE PROYECTOS DE INVESTIGACIÓN AGROPECUARIA

\begin{tabular}{|c|c|}
\hline ACTORES & PERCEPCIONES \\
\hline \multirow{3}{*}{ Estudiantes } & "Son pocos los proyectos en el cucsur, que van dirigidos al desarrollo de la agricultura, campesina". \\
\hline & "A la gran mayoría de los investigadores conocidos, no les interesa hacer proyectos con la gente". \\
\hline & $\begin{array}{l}\text { "El curso de agricultura orgánica que se da en IRNA, es el único en el CUCSUR, que se preocupa por el } \\
\text { rescate y desarrollo de la agricultura campesina". }\end{array}$ \\
\hline \multirow{5}{*}{$\begin{array}{l}\text { Campesinos/ } \\
\text { campesinas }\end{array}$} & $\begin{array}{l}\text { "Se necesita mucha asesoría, para resolver las necesidades que se tienen en las parcelas...son pocos } \\
\text { los maestros del cucsur que salen al campo". }\end{array}$ \\
\hline & "Se necesitan proyectos para disminuir el uso de (agro) químicos tóxicos a los cultivos y al medio ambiente". \\
\hline & $\begin{array}{l}\text { "La región es muy pobre...los proyectos de mejora de la agricultura que se desarrollen aquí, tiene que } \\
\text { tener en cuenta eso... }\end{array}$ \\
\hline & $\begin{array}{l}\text { "Que el cucsuR presente un programa para que el ayuntamiento prohíba los químicos que hacen daño, } \\
\text { y para motivar a los asistentes de estos talleres de agricultura orgánica que son muy buenos". }\end{array}$ \\
\hline & $\begin{array}{l}\text { "En las reuniones donde se presentan convocatorias para proyectos, no nos invitan y si nos invitan, no } \\
\text { entendemos nada, no son para el campesino". }\end{array}$ \\
\hline
\end{tabular}

Las personas productoras y estudiantes vislumbran el gran potencial que podría tener el Centro Universitario para desarrollar proyectos de vinculación en la región, relacionado con el mejoramiento de la agricultura. Sin embargo, se indica que no están enfocados a la resolución de problemas del ambiente rural, sino más bien a otros fines. Esto podría estar relacionado con los problemas de comunicación que se generan entre especialistas y productores, lo cual se presenta a continuación en la tabla 4 . 
Tabla 4

Distanciamiento entre el cuerpo de conocimientos de las personas ingenieras y la población campesina

\begin{tabular}{|c|c|}
\hline ACTORES & PERCEPCIONES \\
\hline \multirow{3}{*}{ Estudiantes } & $\begin{array}{l}\text { "Los conceptos agronómicos que se enseñan en este curso, no son difíciles para explicarlo a los } \\
\text { campesinos, en otras materias si son complejos". }\end{array}$ \\
\hline & $\begin{array}{l}\text { "La primera vez que ingrese a la universidad, sentía miedo, se siente extraño; muchos de los } \\
\text { profesores, secretarias y administrativos se portan como que son de otro mundo". }\end{array}$ \\
\hline & $\begin{array}{l}\text { "Desde que se entra a la escuela, empiezas a educarte con conceptos científicos, cuando te vuelves } \\
\text { ingeniero eres todo un científico,... difícil de que te comprendan los campesinos". }\end{array}$ \\
\hline \multirow{3}{*}{$\begin{array}{l}\text { Campesinos/ } \\
\text { campesinas }\end{array}$} & $\begin{array}{l}\text { "Los ingenieros no saben pasar sus conocimientos a los campesinos..., explican con muchas palabras } \\
\text { rebuscadas y sonantes, el campesino solo escucha pero no entiende". }\end{array}$ \\
\hline & $\begin{array}{l}\text { "El color de la tierra, el suelo, las enfermedades, se nombran diferente..., por ejemplo se le llama } \\
\text { topure a la tierra negra, calichosas a tierras alcalinas y a los ingenieros les enseñan que se llaman de } \\
\text { otra forma, pero luego no saben cómo les nombra la gente del campo". }\end{array}$ \\
\hline & $\begin{array}{l}\text { "Desafortunadamente la escuela no empieza a educar a los muchachos tomando en cuenta lo que les } \\
\text { hemos enseñado, empiezan de cero, y los hacen que se les olviden los saberes de tradición". }\end{array}$ \\
\hline
\end{tabular}

En la tabla 4, se evidencia la gran brecha que existe entre el o la profesionista agrícola y la población campesina, causante de esto son los tecnicismos utilizados: el estereotipo típico del ingeniero que contrasta con el hombre de campo; el elitismo académico, lo cual genera serios conflictos de comunicación y de desconfianza, Esto quizá influya en las percepciones sobre: ¿ccuál podría ser la utilidad práctica de los conocimientos hacia la agricultura que se generan en el cucsur? (tabla 5). 
Tabla 5

Utilidad de los conocimientos e información generada en el CUCSUR

para las actividades agrícolas de la región

VARIABLE: EL CUCSUR COMO ESPACIO DE GENERACIÓN DE CONOCIMIENTOS E INFORMACIÓN

PARA LA AGRICULTURA

\begin{tabular}{|c|c|}
\hline ACTORES & PERCEPCIONES \\
\hline \multirow{3}{*}{ Estudiantes } & $\begin{array}{l}\text { "El contenido de los cursos de agricultura aquí del cucsur son muy buenos, tienen diferencias por } \\
\text { ejemplo este (cursos de agricultura orgánica), y el de agronomía. El de agronomía, es muy complejo } \\
\text { para que lo entiendan los productores, pues no es para ellos, es para formar ingenieros". }\end{array}$ \\
\hline & $\begin{array}{l}\text { "Las publicaciones que se hacen son de mucha calidad, pero el problema es que a veces ni uno las } \\
\text { entiende, esta información no pueden leerla los campesinos". }\end{array}$ \\
\hline & $\begin{array}{l}\text { "Se genera mucha información pero lo difícil es bajarla al nivel de comprensión de los campesinos, o } \\
\text { sea casi no hay profes o doctores que se preocupen por eso, ellos solo publican y ya...". }\end{array}$ \\
\hline \multirow[b]{2}{*}{$\begin{array}{l}\text { Campesinos } \\
\text { /campesinas }\end{array}$} & $\begin{array}{l}\text { "No hay duda que lo que se publique, o lo que se enseñe aquí tenga mucha calidad, el ejemplo está en } \\
\text { estos cursos... el problema es cómo enseñarnos con lenguaje sencillo, todo lo que se dice aquí (en los } \\
\text { cursos-talleres), se puede comprender y lo está hablando un ingeniero, un maestro". }\end{array}$ \\
\hline & $\begin{array}{l}\text { "Se necesitan libros y folletos de agricultura que puedan ser leídos, con letras grandes..., dibujos, } \\
\text { recomendaciones que pueda entenderlas cualquier compañero". } \\
\text { "Debe de ser igual con los congresos que se hacen aquí, estos no se hacen para los } \\
\text { campesinos, cuando nos invitan se siento raro, tanta gente que sabe y uno nada más mirando, } \\
\text { sin entender nada...". }\end{array}$ \\
\hline
\end{tabular}

La tabla 5 contiene críticas que ponen en debate el contenido del conocimiento que se genera con utilidad práctica para el desarrollo de la agricultura en el cucsur. Aunque ambas partes consideran que se genera información de alta calidad, el problema es hacerlo comprensi- ble para los principales actores de la agricultura. Esto tiene relación con lo que se presenta en el siguiente apartado respecto al papel que tiene el cucsur en la formación de profesionistas que puedan responder a las necesidades campesinas de la región (tabla 6 ). 
Tabla 6

Percepciones sobre la formación de profesionistas que respondan a las necesidades de la agricultura campesina en la región

\begin{tabular}{|c|c|}
\hline ACTORES & PERCEPCIONES \\
\hline \multirow{4}{*}{ Estudiantes } & $\begin{array}{l}\text { "Si hay cursos y programas muy buenos, este por ejemplo, y se aprenden, buenas cosas de } \\
\text { agricultura orgánica... pero el buen trabajo y el bien pagado lo tienen las empresas de agroquímicos } \\
\text { o de semillas como Bayer, Dupont, Monsanto". }\end{array}$ \\
\hline & $\begin{array}{l}\text { "Dicen que los que viene a estudiar ya no regresan a sus lugares de origen, porque lo que se aprende } \\
\text { aquí..., solo sirve para trabajar en empresas de agroquímicos o en invernaderos". }\end{array}$ \\
\hline & $\begin{array}{l}\text { "Allá afuera es otra cosa, muchos ingenieros que tomaron los cursos de agricultura orgánica, andan } \\
\text { trabajando en los invernaderos o en esas empresas". }\end{array}$ \\
\hline & $\begin{array}{l}\text { "Si hay cursos y programas para desarrollar la agricultura, pero la mayoría es para la agricultura } \\
\text { empresarial... afuera tiene mucho peso, son empresas muy poderosas". }\end{array}$ \\
\hline \multirow{3}{*}{ Campesinos } & $\begin{array}{l}\text { "Cuando los muchachos regresan al rancho o al pueblo como ingenieros quieren trabajos bien } \\
\text { pagados, en el rancho no hay quien te pague bien, solo las empresas o los viveros". }\end{array}$ \\
\hline & $\begin{array}{l}\text { "A diferencia de este curso..., a los muchachos se les educa para el desarrollo de la agricultura } \\
\text { industrial, no para desarrollar la agricultura del campesino". }\end{array}$ \\
\hline & $\begin{array}{l}\text { "El único programa que conocido es este, pero hoy ingenieros salidos de aquí, que no saben hacer } \\
\text { nada de lo que se ve en este curso..., andan tirando y aplicando puros venenos". }\end{array}$ \\
\hline
\end{tabular}

En la tabla 6, se evidencia que hay un desfase entre lo que se enseña de agricultura orgánica en el cucsur y la agricultura empresarial - la cual se vincula con las necesidades laborales del entorno-. En este sentido, como la agricultura se encuentra influenciada por la agricultura empresarial, esta no contiene elementos para la revalorización de las prácticas y los saberes locales del campesinado (tabla 7).

\section{C) SABERES Y PRÁCTICAS LOCALES}

La tabla 7 dimensiona los grandes problemas que tiene la academia para valorizar, incluir y complementar los conocimientos y los saberes locales, es decir, se encuentran desvinculadas de las cuestiones técnicas. 
Tabla 7

Prácticas, saberes locales y factores causantes de desplazamiento

\section{VARIABLE: PRÁCTICAS Y SABERES LOCALES}

\begin{tabular}{|c|c|}
\hline ACTORES & PERCEPCIONES \\
\hline \multirow{5}{*}{ Estudiantes } & $\begin{array}{l}\text { "Yo que vengo de rancho, se hacer muchas cosas del campo..., muchos profesores no saben } \\
\text { hacerlas, pero no me atrevo a decirle al maestro que así no se hace, o que está mal, por temor a } \\
\text { reprobar, él es el que califica". }\end{array}$ \\
\hline & $\begin{array}{l}\text { "En las clases son pocos los maestros que dejan expresar los conocimientos recibidos de los } \\
\text { padres y abuelos... se reciben regaños por usar las palabras con las que se nombran las cosas en } \\
\text { el rancho". }\end{array}$ \\
\hline & $\begin{array}{l}\text { Hay compañeros que saben hacer muchas cosas, ...es impresionante..., dicen que todo esto que } \\
\text { saben, lo aprendieron de sus tíos, los abuelos y los papás". }\end{array}$ \\
\hline & $\begin{array}{l}\text { "Si te expresas diferente, (los profesores), te llaman la atención y luego te preguntan que si tienes } \\
\text { sustento científico sobre lo que dices". }\end{array}$ \\
\hline & $\begin{array}{l}\text { "Los saberes locales se pierden aquí, por ejemplo muchos de los que viven en comunidades } \\
\text { indígenas, no se expresan igual aquí que allá, aquí hasta te hacen cambiar el acento y las } \\
\text { palabras, ya ni se diga de explicar a los profes como perciben los recursos y la vida de los padres y } \\
\text { abuelos, no solo los compañeros se burlan también algunos maestros". }\end{array}$ \\
\hline \multirow{5}{*}{$\begin{array}{l}\text { Campesinos/ } \\
\text { campesinas }\end{array}$} & $\begin{array}{l}\text { "Nosotros mismos les decimos a los hijos que es malo quedarse sin estudiar, así como nos } \\
\text { quedamos nosotros, ahí se empieza a negar saberes, ahí se empieza a renegar de lo que somos". }\end{array}$ \\
\hline & $\begin{array}{l}\text { "Es común escuchar de muchachos del rancho que..., muchos maestros que andan enseñando } \\
\text { agricultura, no saben ni agarrar la pala o el azadón". }\end{array}$ \\
\hline & $\begin{array}{l}\text { "La práctica y los saberes me han hecho a mí..., me atrevo a decir que en mi parcela no hay } \\
\text { ningún ingeniero que me diga cómo hacer las cosas, tengo más de } 40 \text { años conociéndola". }\end{array}$ \\
\hline & $\begin{array}{l}\text { "Si..., se aprenden cosas buenas en la universidad, pero lo malo es que les quitan lo pocos } \\
\text { saberes, que aprendieron con nosotros". }\end{array}$ \\
\hline & $\begin{array}{l}\text { "Es importante que enseñen a los ingenieros a respetar las prácticas de los campesinos, nosotros } \\
\text { las hemos desarrollado por toda la vida, ellos estudian solo cuatro años". }\end{array}$ \\
\hline
\end{tabular}

\section{D) LA TIERRA COMO EXPRESIÓN CULTURAL}

En este apartado se analiza el componente de las expresiones culturales, para lo cual se presentan las percepciones respecto a la variable tierra (tabla 8). 
Tabla 8

Percepciones sobre la tierra como espacio productivo y expresión cultural

Grupo autoseleccionado participante de la Escuela Campesina

Centro Universitario de la Costa Sur

Universidad de Guadalajara, 2010-2019

\begin{tabular}{|c|c|}
\hline ACTORES & PERCEPCIONES \\
\hline \multirow{5}{*}{ Estudiantes } & $\begin{array}{l}\text { "Bueno, la tierra es vista como lo es una madre, inclusive que nos hicieron de tierra, de barro, de } \\
\text { lodo, pero ya para la universidad tenemos que referirnos de otra manera a la tierra y los recursos, } \\
\text { si no imagínese la regañada de los profes". }\end{array}$ \\
\hline & "Es el lugar donde convive la fauna silvestre y el hombre". \\
\hline & "El lugar donde habita el ser humano y convive con los recursos naturales". \\
\hline & "Es la tierra la que brinda la vida, genera recursos que pueden terminarse si no se utilizan bien". \\
\hline & "La tierra genera recursos naturales, alimento, vestido y también servicios ambientales". \\
\hline \multirow{5}{*}{$\begin{array}{l}\text { Campesinos/ } \\
\text { campesinas }\end{array}$} & "La tierra es la que da todo, de ahí nace, ahí se pasa toda la vida y ahí se terminará". \\
\hline & "Sin tierra no se es nada..., ella da la vida". \\
\hline & $\begin{array}{l}\text { "La tierra está enferma, se tiene que limpiar para seguir viviendo..., los mineros, los tala montes, } \\
\text { ahora los aguacateros no ven como están destruyendo y llenando de químicos el agua y la tierra, } \\
\text { ¿Que van ellos a tomar y a comer con todos sus millones, la misma comida y agua que ellos están } \\
\text { envenenando?". }\end{array}$ \\
\hline & $\begin{array}{l}\text { "Sin tierra las familias se descomponen y muchas terminan mal..., en el rancho hay muchos } \\
\text { casos de esos, gente que vendió sus tierras y acabaron de limosneros, la tierra no tiene precio". }\end{array}$ \\
\hline & $\begin{array}{l}\text { "La tierra es el corazón de la familia, sin tierra las familias se desintegran y se pierden muchos de } \\
\text { sus valores". }\end{array}$ \\
\hline
\end{tabular}

Existen diferencias poco notorias sobre las percepciones que tienen las personas campesinas y estudiantes. Estos últimos integran en su discurso el concepto recurso y perciben a la tierra como generadora de bienes y servicios, mientras que los campesinos desarrollan más el significado cultural empírico ligado a la cosmovisión indígena.

\section{E) VALORIZACIÓN DE LOS SABERES EN LOS CURSOS DE AGRICULTURA}

Respecto a los saberes campesinos y su valorización en los cursos de agricultura, se encontró que en las técnicas mostradas en los talleres campesinos, la gran mayoría de los productores afirman reconocer una o más prácticas de las que se presentan o elaboran en el curso de agricultura orgánica. Es decir, que ya forman (o han formado) parte de sus saberes campesinos. Sin embargo, más del $50 \%$ de los estudiantes reconoce algún uso de las técnicas mencionadas; para ejemplificarlo se muestran algunos de estos en la tabla 9 . 
Tabla 9

Saberes campesinos y técnicas de agricultura orgánica

Percepciones del grupo autoseleccionado participante de la Escuela Campesina

Centro Universitario de la Costa Sur

Universidad de Guadalajara, 2010-2019

\begin{tabular}{|c|c|}
\hline TÉCNICA DE ENSEÑANZA & SABERES CAMPESINOS SOBRE LA TÉCNICA \\
\hline Uso de estiércol de vaca como fertilizantes & $\begin{array}{l}\text { "Siempre se había utilizado estiércoles hasta que llegaron los fertilizantes". } \\
\text { "De papá aprendí a quemar los estiércoles para que no dañar a la planta". } \\
\text { "Algunas "mierdas" son más fuertes, como las de borrego, gallina, conejo, } \\
\text { murciélago". } \\
\text { "No se deben de echar frescas se deben de dejar fermentar". }\end{array}$ \\
\hline Minerales, cenizas y harinas de hueso & $\begin{array}{l}\text { "Los abuelos y los padres quemaban los coamiles para sacar los minerales } \\
\text { de las cenizas". } \\
\text { "Ya se utilizaba las cenizas para controlar las hormigas y al hongo que le } \\
\text { dicen de "Chauixtle". } \\
\text { "Las vacas y los venados encuentran los minerales en los "terreros", de ahí } \\
\text { los lamen, eso quiere decir que en el cerro están los minerales". }\end{array}$ \\
\hline Minerales, cenizas y harinas de hueso & $\begin{array}{l}\text { "Los minerales son importantes..., una planta o animal sin minerales no } \\
\text { se desarrolla bien". }\end{array}$ \\
\hline Suero de leche y melazas & $\begin{array}{l}\text { "Los sueros han sido siempre utilizados para engordar puercos". } \\
\text { "Los sueros tienen mucha energía, igual que las melazas". } \\
\text { "Cuando había pudrición de la mala, los abuelos echaban suero y melaza al } \\
\text { suelo y se controlaba". }\end{array}$ \\
\hline Azufre y Cobre & $\begin{array}{l}\text { "Fueron los primeros fungicidas en aprenderse a hacer". } \\
\text { "No eran caros y con eso se controlan muchos hongos de los cultivos". } \\
\text { "Son fáciles y baratos de hacer, muchos que se dedican a la agricultura los } \\
\text { hacen más de una vez". }\end{array}$ \\
\hline Tierra de hoja y materia orgánica & $\begin{array}{l}\text { Los suelos de antes no necesitaban fertilizantes porque tenían mucha } \\
\text { tierra topure o negra. } \\
\text { "De chico observaba que mi padre desviaba el rio para que metiera } \\
\text { "lamadal"... (tierra de hoja), y la tierra se fertilizaba". } \\
\text { En los corrales donde se pudre la tierra de hoja, las plantas se dan solas, no } \\
\text { necesita ser abonarlas. }\end{array}$ \\
\hline
\end{tabular}

\section{DISCUSIÓN}

De aproximadamente 200 productores que acudieron a los talleres campesinos en el Cucsur, entre 8-10 de ellos continúan asistiendo con regularidad a todos los cursos. No todos son del municipio de Autlán de Navarro, y los rangos de tiempo para llegar a los cursos oscilan entre veinte minutos $y$ tres horas aproximadamente. Lo anterior significa que existe un gasto significativo en tiempo y combustible para el transporte que corre a cargo de las personas interesadas, lo cual indica que los productores que asisten de forma regular tienen un verdadero interés en dichos cursos.

Es importante remarcar que estos productores han impartido charlas de actualización disciplinar para estudiantes de nuevo ingreso en los talleres de agricultura orgánica (estudiantes y productores), así como de establecer fincas de producción orgánica basados en la aplicación de 
los nuevos conocimientos, habilidades y capacidades obtenidas en dicho curso.

Lo anterior resulta relevante considerando que la mayoría no tienen educación media ni superior; aun así, han acompañado como instructores $y$ facilitadores de esta experiencia que se ha extendido a más de 500 productores de la Región Sur, Costa Sur y Sierra de Amula en el Occidente de Jalisco. Estos productores han integrado alguna práctica de agricultura orgánica en sus parcelas basados en la divulgación de estos conocimientos.

Pero, ¿cómo es que los campesinos han podido comunicar el contenido de los temas $y$ de los cursos que se desarrollan en los módulos de agricultura orgánica a otros productores de la región, sin contar con los conocimientos propios de los universitarios? En este proyecto se identifica algunos factores que hicieron posible lo anterior. Entre estos se vislumbra que los campesinos se muestran capaces de entender la problemática local, porque viven continuamente en ella; no de forma teórica, como convencionalmente se enseña.

Además, tienen la capacidad de proponer alternativas basadas en la propia experiencia, las cuales son fortalecidas por las mismas estrategias de educación popular que integran los talleres de agricultura orgánica.

Asimismo, se observa que los conocimientos campesinos que se transmiten de forma oral en la comunidad, es una forma de comprender los problemas de forma común, de igual manera, la defensa de la tierra, no se percibe solo como una lucha por disminuir el deterioro de un recurso, sino que es vista como "la madre que da la vida", permite conformarlos como individuos que participan en el desarrollo de políticas alternativas propias $y$ de generar mecanismos de resistencia y construcción de alternativas sociales (Díaz, 2007).

Por eso, cuando se habla del rol que tienen los talleres de agricultura orgánica, por un lado, se refleja en el contenido de los temas, $y$ por otro lado, resulta interesante entender la función de la histórica palabra campesina como símbolo de lucha social, que se vuelcan a entender el sentido de las clases oprimidas versus las clases dominantes, tal y como lo menciona
Paulo Freire (2005), en su excelsa obra de la $P e$ dagogía del Oprimido:

(...) es impresionante observar como en las primeras alteraciones de una situación opresora, se verifica una transformación en esta auto-desvalorización (...). Nos decían que no producíamos porque éramos borrachos, perezosos. Toda mentira, ahora que somos respetados como hombres vamos a demostrar a todos que nunca fuimos "borrachos" ni perezosos. Éramos explotados eso sí (...). (p. 105).

La cita anterior conduce a reflexionar sobre la percepción histórica de la clase dominante sobre los campesinos y sus saberes. Particularmente, lleva a considerar el hecho de que dicha percepción no es compartida ni por los campesinos, ni por los participantes en los talleres de agricultura orgánica.

\section{A) VINCULACIÓN UNIVERSIDAD-CAMPESINO}

Las percepciones de los productores evidencian que la imposición de un modelo educativo único afecta de manera sustancial la vinculación universidad-sociedad. En este caso, los productores reconocen a los talleres de agricultura orgánica como espacios abiertos para el desarrollo de nuevas plataformas de vinculación cucsuR-problemática campesina.

Ellos exigen otras formas de generar, difundir y utilizar el conocimiento técnico $y$ científico que se genera en el CUCSUR, lo cual concuerda con lo establecido por Escalante et al. (2009). Sin embargo, el hecho de ser considerados (como ellos lo vislumbran) como los "otros", los diferentes, es una traba cultural que no puede hacer posible que el especialista o el académico logre inmiscuirse en "la otra comunicación".

Por lo tanto, los proyectos de vinculación que requieren los campesinos no pueden ser aquellos que promueven el conocimiento académico como dominante y excluyen conceptos, ideas, códigos, saberes locales y símbolos de la identidad campesina.

De esta manera, es importante señalar que el modo científico y técnico con el que se 
generan conocimientos en las aulas (con el cual se educa a los estudiantes) no debe desplazar los conocimientos campesinos que traen de sus comunidades, sus experiencias, sus vivencias $y$ su cosmovisión. Hacerlo podría generar un distanciamiento entre el cuerpo de conocimientos teóricos y empíricos, aun cuando ya han dejado la universidad y regresado a sus comunidades.

Se concluye este apartado señalando que las acciones de vinculación como los talleres de agricultura orgánica pueden crear puentes para unir los conocimientos teóricos de la "academia" a los conocimientos campesinos. Consecutivamente, esto servirá para detonar acciones tanto de emprendimiento de proyectos de desarrollo rural, como del componente sociocultural.

\section{B) TRANSFERENCIA DE TECNOLOGÍAS Y DIFUSIÓN DE LA INFORMACIÓN}

El revalorizar las percepciones campesinas que reconocen que la universidad tiene las capacidades tecnológicas para enfrentar la problemática de la región es importante. La mayoría de los participantes de los talleres hizo fuertes críticas sobre los mecanismos de difusión y divulgación científica que tiene la institución (a diferencia de los talleres de agricultura orgánica). Por lo tanto, cabe discutir los puntos que derivan de este tema. Primero, destacar que los campesinos perciben que los libros, artículos, eventos académicos como congresos, foros y talleres -entre otros- están hechos para especialistas. En este mismo tenor, no se integran estrategias de comunicación para involucrar a la población campesina.

De esta manera se confirma que la experiencia generada en los talleres campesinos aporta elementos para proponer estos espacios. Por ejemplo, considerar la creación de plataformas de generación y difusión de información técnica-científica que pueda ser comprensible para las personas campesinas $y$, al mismo tiempo, útil para fortalecer sus experiencias y conocimientos.

En esta nueva relación, la persona campesina es capaz de comprender, repetir y darle significado a los conceptos que históricamente le habían sido ocultados. Entonces, comienza a entender e interpretar los discursos de las personas profesionistas del campo que eran ininteligibles, a la vez que comienza a mejorar su situación de vulnerabilidad, de manipulación y dependencia tecnológica que actualmente ocurre (Morales y Alatorre, 1994).

\section{C) SABERES CAMPESINOS Y LA CONCEPCIÓN CULTURAL DE LA TIERRA}

Cuando los participantes señalan que el uso del trabajo por las personas campesinas en las diferentes condiciones o ciclos naturales son los que les permiten interpretar la tierra de forma diferente a los profesionistas o académicos, queda claro que existen dos dimensiones para referirse a esta $y$ a sus los recursos naturales.

Mientras las personas campesinas aseguran que la tierra es el bastión para la vida y para la enseñanza, los y las estudiantes que llegan a la universidad cambian esta forma de pensar para hacerlo de manera más científica, es decir, menos centrada en los no reconocidos saberes populares. En este sentido, los saberes que habían sido adquiridos junto a sus mayores $y$ mediante las experiencias se transforma de manera radical al ir integrando conocimientos del funcionamiento ecológico científico de la naturaleza.

Las experiencias $y$ vivencias ancestrales se excluyen automáticamente para dar lugar a la enseñanza mecanicista, autoritaria $y$ lineal que exige la formación disciplinar de la universidad, tal y como lo señala un campesino:

Cuando llega de la escuela le digo (a mi hijo), vente pues... vamos a resolver tal o cual problema que tenemos ya sea con el ganado, ya sea con la milpa, la caña, y ¿qué es lo que me dice?, es que en la escuela nos enseñan otras cosas diferentes, cosas más de ingeniero. (Participante Escuela Campesina, comunicación personal, 2019),

Por supuesto que esta ruptura es vista como un comportamiento negativo entre ambos participantes de los talleres (estudiantes y campesinos). 
En cuanto a los estudiantes, se les reeduca con la percepción de que los conocimientos académicos son los únicos válidos. Por lo tanto, las enseñanzas de la "gente del campo" adquieren una connotación de ignorancia, supersticiones $y$ "ocurrencias", entonces esto se vuelve motivo de vergüenza.

Por esta razón, cabe rescatar que en los talleres de agricultura orgánica no se niegan esos saberes, esas emociones y vivencias. De este modo, el analizar, vivir, experimentar, comprender y conocer el entorno debe de hacerse de una forma integral, holística y multidimensional (Delgado y Rist, 2016).

En este mismo apartado, se destaca que el involucramiento de los saberes locales se ha considerado desde hace varios años como una estrategia de los movimientos de vanguardia para el desarrollo del campo y de la educación popular campesina, como uno de los ejes principales que conlleva al empoderamiento de la sociedad.

El conocimiento popular se nutre desde siempre de los "saberes locales". Estos saberes que tienen como sentido (como lo menciona el texto anterior), liberarse de lo que los tiene prisioneros, así como entender el contexto desde donde surge el despojo y la destrucción de su territorio, y el saqueo de sus recursos. Todos estos son elementos fundamentales para trazar los caminos y las guías que los llevan a defender su identidad y territorio, visto como la cuna donde se genera y reproduce la vida del campesino y su familia (Roncal, 2017).

\section{CONCLUSIONES}

La modalidad en que se desarrollaron los talleres de agricultura orgánica en el cucSUR, resultó fundamental para comprender las percepciones de los participantes respecto a las diversas formas posibles de generar y enriquecer el conocimiento de las ciencias agropecuarias para lograr procesos más sustentables de producción. Asimismo, se reconoce que las personas campesinas participantes tienen saberes y prácticas locales, con lo que al integrarlos a la dinámica educativa de los talleres son capaces de fortalecer espacios educativos como los mencionados.
De aquí se deriva la importancia de generar y recuperar espacios educativos para la participación de esta población en metodologías alternativas de enseñanza. Se sostiene que la formación de un pensamiento crítico de la educación transformadora de los espacios socioproductivos de México se construye mediante el compartir saberes, experiencias, creencias y verdades que rebasan los escalones del mundo académico privilegiado.

Con estos indicadores es posible (arriesgarse a) señalar que estas nuevas modalidades de generación y comunicación del conocimiento que se desarrollan en los talleres de agricultura orgánica podrán fungir como instrumentos de acción para la promoción de un modelo educativo que permita dinamizar al campo y a la agricultura en el intento de romper los esquemas hegemónicos de la educación agronómica.

Sin embargo, debido a que la presente investigación se orientó en la integración de los saberes campesinos en la agricultura, es necesario profundizar en el análisis de otros factores que pudieron quedar excluidos, como las percepciones de los académicos sobre la misma temática.

Además, se considera que es necesario erradicar primero de las universidades esa práctica social de desvalorización del campesino mexicano y sus saberes. Dejar ir la noción histórica que construyó la figura del campesino, pobre, desarrapado, de huarache y sombrero, que resultó en la desvalorización de estos individuos, percibidos como incapaces e ignorantes, sin poder generar conocimiento.

Finalmente, las aportaciones de este trabajo contribuyen a enriquecer la información existente sobre las metodologías educativas agropecuarias, así como para evidenciar las diferencias y contradicciones que existen entre la agricultura campesina con la agricultura empresarial en el contexto de la enseñanza universitaria.

\section{REFERENCIAS}

Calva, J. L. (1993). El Modelo de desarrollo agropecuario impulsado mediante la ley agraria y el TLC. En Calva J. L. (coord.), Alternativa para el campo mexicano (pp. 15-43). Ed. Fontamara. 
De la Cuadra, F. (2015). Buen Vivir: ¿Una auténtica alternativa post-capitalista? Polis Revista Latinoamericana. http://journals.openedition.org/polis/10893

Delgado, F. y Rist, S. (2016). Las ciencias desde la perspectiva del diálogo de saberes, la transdiciplinareidad y el diálogo intercientífico. En Delgado, F. y Rist, S. (Ed.), Ciencias, diálogo de saberes e interdisciplinariedad: Aportes teóricos metodológicos para la sustentabilidad alimentaria $y$ del desarrollo (pp. 35-60). AGRUCO.

De Sousa Santos, B. (2010). Descolonizar el saber, reinventar el poder. Montevideo Uruguay: Ed. Trilce.

Díaz, P. L. (2007). Tierra y Educación en el campesinado de Santiago del Estero. Nuestra América.

Figueroa, B. P., Gerritsen, P. R. W., Villalvazo, V. M. y Cruz, G. (2005). Articulando la sostenibilidad ecológica, económica y social: el caso del cacahuate orgánico. Economía, Sociedad y Territorio, v (19), 447-497.

Freire, P. (2005). Pedagogía del Oprimido. Siglo XXI.

Gerritsen, P.R.W., Cruz, G., Villalvazo, V. y Figueroa, P. (2004). Productos regionales en el Occidente de México: ¿respuestas locales frente a la globalización económica? [Ponencia]. Congreso Internacional Agro-empresas rurales y Territorio (ARTE), Toluca, Estado de México.

Gerritsen, P.R.W., Villalvazo, V.M., Figueroa, B. P. y Cruz, G. (2012). Fortalecimiento de procesos endógenos en la Costa Sur de Jalisco. Imágenes de una nueva ruralidad emergente. En Gerritsen, P.R.W. (Coordinador), Sociología rural y medio ambiente. Tópicos selectos de Jalisco (pp. 50-70). Plaza y Valdés/
Editorial Universitaria/Universidad de Guadalajara.

Kaplun, M. (1985). El Comunicador Personal. CIESPAL.

Levin, J. (1992). Fundamentos de estadística en la investigación social. ( $2^{\mathrm{a}}$. Edición). HARLA.

Loera-González, J. J. (2015). La construcción de los buenos vivires; entre los márgenes y tensiones ontológicas. Polis Revista Latinoamericana. http://journals.openedition.org/polis/10654.

Morales, J. y Alatorre, F. (1994). La política agropecuaria en México (1988-1994). Renglones (28), 48-54. ITESO.

Núñez, C. H. (1985). Educar para transformar, transformar para educar. IMDEC A.C.

Roncal, P. (2017). El vivir bien como alternativa al desarrollo y no como modelo de desarrollo alternativo. En Gerritsen, P., Rist S., Morales, H.J., y Tapia, P., (Ed.), Multifuncionalidad, Sustentabilidad y Buen Vivir. Universidad de Guadalajara.

Saxe-Fernández, J. (1999). Globalización e Imperialismo. En Saxe-Fernández (coord.), Globalización, crítica a un paradigma (pp. 9-68). Plaza y Janéz.

Toledo, V. M., y Barrera-Bassols, N. (2008). La memoria biocultural: la importancia ecológica de las sabidurías tradicionales. Icaria editorial.

Villalvazo-López, V. M., Gerritsen, P., FigueroaBautista, P. y Cruz-Sandoval, G. (2003). Reforzando el desarrollo endógeno en el occidente de México. Algunas reflexiones teóricas-prácticas. Sociedades Rurales. Producción y Medio Ambiente, 4(1), 41-50.

Fecha de ingreso: 03/02/2020 Fecha de aprobación: 13/09/2021 